\title{
REVISTA DE REVISTAS. LIBROS. COMENTARIOS
}

\section{INTRODUCTORY MICOLOGY}

A. Alexopoulos, C.J. y Mins, C.W. 3era. Edicion. John Wiley and Sons Inc. New York,1979. 632 páginas. Precio US\$ 24,95.

Si el comentario de la 2a. edición (1962)era óptimo, la actual no puede menos de ser calificada como excelente, ya que realmente incluye una amplia y moderna renovación en todos sus capítulos donde el especialista $y$ el estudiante encontrarán una rica información en las áreas de la taxonomía y morfología fúngica complementada con nuevas fotografías y una amplia y renovada bibliografía de gran utilidad para el micólogo. Escrito en forma clara y amena, interesa desde el principio.

Los cambios taxonómicos en todos los grupos son importantes y reflejan los claros conceptos de los autores con los avances en los diferentes campos; notable es el tratamiento de los Ascomycetes bajo el concepto "Luttrelliano". En los hongos imperfectos, mantiene una línea moderada ante los conflictivos estudios de éstos a un nivel específico.

Toda persona dedicada a esta ciencia biológica deberá contar en su biblioteca personal con esta publicación que servirá de apoyo y consulta para todo curso de especialización en el ramo.

\section{ZOONOSES AND}

TRANSMISSIBLE DISEASES COMMON TO MAN AND ANIMALS

Acha, P.N.; Szyfres, B.

Washington, D.C.; Panamerican

Health Organization (1977) XVIII

+ 708 páginas (Español). Precio

US\$ 15.00.-

La Sección correspondiente a las Micosis (pag. 131-166) considera los siguientes aspectos: etiología, distribución geográfica, presencia y sintomas en hombre y animales, fuentes de infección y mecanismos de transmisión, rol epidemiológico de los animales, diagnóstico y control en los capítulos que se mencionan a continuación: Actinomicosis, Adiaspiromicosis, Aspergilosis, Blastomicosis, Candidosis, Coccidioidomicosis, Criptococosis, Dermatofitosis, Esporotricosis, Histoplasmosis, Maduromicosis, Nocardiosis, Rinosporidiosis y Zigomicosis. Es una obra de indudable valor por su extensión y la actualización de los temas tratados.

\section{THE GENUS PENICILLIUM AND ITS TELEOMORPHIC STATES EUPENICILLIUM AND TALAROMYCES.}

\section{John I. Pitt.}

Academic Press, London, 1979. $v i+634$ páginas. Precio US\$92,00.

Es un manual importante en la clasificación del género, porque en primer lugar actualiza los conceptos morfológicos, fisiológicos y taxonómicos del género Penicillium y géneros afines. Su utilidad práctica deriva hacia la industria y la ecología del género en el suelo $y$ en otros ambientes. El autor divide a éste, aplicando los conceptos de crecimiento, temperatura y su afinidad en relación al agua. Además considera sus estados teleomórficos en Eupenicillium y Talaromyces. Presenta una extensa revisión de la nomenclatura, sin embargo el empleo de su metodología basada ya sea en el crecimiento $y$ otras en la morfologia implica el uso de gran cantidad de material que dificulta el rápido acceso a la determinación de las especies. Carece de las minuciosas descripciones del "Manual of the Penicillia" K.B. Raper y C. Thom (1949) y la identificación de una determinada especie no es fácil para el principiante. A pesar de ello es innegable su valor para el especialista, siempre que se complemente con otras literaturas especializadas en el género.

A nuestro parecer sigue siendo el antiguo "Manual of the Penicillia (1949) Raper y Thom un texto de consulta básico que no puede relegarse definitivamente aún, a un segundo plano.

\section{INTRODUCTION TO MODERN MYCOLOGY \\ J.W. Deacon. \\ Halstead Press, John Wiley \& Sons, New York, Toronto, 1980. \\ (Basic Microbiology, vol. 7) 197 \\ páginas. Precio US\$18,95.}

A pesar de ser un libro de pocas páginas, introduce conceptos modernos sobre Fisiología, Bioquimica y Biología Molecular relacionados con la Micología. Es un texto ideal para biólogos que deseen tener una amplia visión del mundo micológico. Sus conceptos son claros y al alcance de todo lector con conocimientos básicos en hongos. Es obvio que por su reducido volumen, no abarca todos los campos de esta ciencia y se hace notoria la brevedad en algurios capítulos como la taxonomía fúngica.

Es un texto recomendado para estudiantes universitarios, instructores y graduados.

\section{APARTADOS. REVISTAS}

Wild Animals as reservoirs of human pathogenic dermatophytes.

L. Ozegovic.

Facultad de Veterinaria, Sarajevo, Univ. Yugoslavia, Yugoslavia Preusser (eds). Medical Mycology.

Zbl. Bakt. Suppl. 8 Gustav Fischer Verlag. Stuttgart, New York, 1980.

Este estudio fue llevado a cabo en animalés de vida libre, silvestres, cautivos en zoológicos y otros utilizados en la industria peletera. Los animales silvestres considerados incluyeron pequeños roedores, portadores de especies del género Trichophyton ( $T$, mentagrophytes var. granulosum, var. erinacei, var. quinckeanum; T. ajelloi y T. terrestre) y en menor proporción especies del género Microsporum (M. persicolor, M. canis, M. gypseum, M. fulvum y M. cookei). Entre los animales silvestres cautivos se incluyeron caninos y felinos, 
con una microflora propia de los animales domésticos (perros y gatos: M. canis y M. gypseum), además de pequeños roedores $(T$. mentagrophytes).

Actualmente los aislamientos de dermatófitos potencialmente patógenos han ido en aumento $(T$. ajelloi, $\boldsymbol{\Upsilon}$. terrestre, M. cookei) fenómeno ampliamente comprobado a través de la inoculación experimental.

El autor concluye que entre los animales más peligrosos para el hombre, entre los mamíferos, están los ratones y las ratas, en tanto que los cobayos no sólo constituyen una fuente de infección para los animales domésticos sino también para los silvestres. Respecto a la micoflora propia de los animales silvestres, el autor estima que podría ser interpretada como un intento de evolución de los dermatofitos desde su habitat primario, el suelo, hacia la patogenicidad en los animales y el hombre En cambio la flora dermatofítica de los animales cautivos podría ser considerada como la proyección de los dermatofitos zoofílicos patógenos adaptados a su nuevo habitat. Sin embargo, es necesario tener presente que los animales silvestres no siempre presentan signos clínicos de las dermatofitosis, los hombres son infectados clínicamente o bien por animales aparentemente sanos.

Los hechos mencionados anteriormente indican la existencia de portadores naturales, condición que se manifestó en los animales analizados y que por lo tanto merecen toda nuestra atención.

\section{SAPROBIC RESERVOIRS OF CRYPTOCOCCUS NEOFORMANS}

Pal, $M$.

(Abstract) Indian Journal of Public Health (1978) 22 (4) 327-328.

En inglés. Med. Mycology Dep.; Vallabhbhai Patel Chest Inst., Univ. Delhi-110007, India.

De un total de 489 muestras (208 excrementos de palomas, 46 excretas de palomas con suelo, 38 excretas de otros pájaros, 53 excretas de murciélagos y 144 suelos) de Delhi y sus alrededores se investigó la presencia de Cryptococcus neoformans. Sólo en 39 aislamientos se encontró Cryptococcus neoformans, 33 corresponden a excrementos de palomas, 5 de excrementos de otros pájaros y 1 de suelo.

\section{THE ECOLOGY OF ATHLETE'S FOOT.}

Ryall, $C$.

New Scientist (1980) 87(1214)

528-530 (Inglés), Polytechnic of

Central London, London, U.K.

Se discuten los factores que condicionan el desarrollo del pie de atleta (Trichophyton mentagrophytes, $T$. interdigitale, $T$. rubrum y Epidermophyton floccosum). Entre los factores abióticos se indican temperatura, $\mathrm{pH}, \mathrm{y}$ razón entre $\mathrm{CO}_{2}$ y O2. Los factores bióticos definidos como aquellos que influyen en forma determinante en la sobrevivencia de los hongos $\mathrm{y}$ otros microorganismos asociados en competencia, destacando la habilidad de los dermatofitos de degradar la queratina proporcionando a partir de este substrato nutrientes más aptos para las bacterias.

El autor estima que la rápida degradación de la piel por acción de los dermatofitos, sólo se desencadena cuando están presentes ambos grupos, bacterias y hongos, hecho que sugiere que las enzimas de los dermatofitos y de las bacterias podrían actuar sinérgicamente.

\section{INMUNITY TO \\ CANDIDA ALBICANS}

Rogers, T.J.; Balish, E. Microbiological Reviews (1980) 44(4)660-682. En inglés, Dep. Microbiol., Oregon State Univ. Corvallis, Ore. 97331 , USA.

Este reporte discute los aportes brindados por la investigación en la comprensión básica de la inmunidad adquirida a innata a Candida albicans. Entre los tópicos analizados están la flora microbiana, factores hormonales, fagocitosis, factores innatos en los sueros, inmunidad adquirida. Se incluyen modelos experimentales, inmunidad mediada por anticuerpos y por células (candidosis mucocutánea y estudios en animales de experimentación) y la histopatología e inmunosupresión determinada por la infección.
THE VALUE OF FUNGAL SURVEILLANCE CULTURES AS PREDICTORS OF SYSTEMIC FUNGAL INFECTIONS

Sandford, G.R.; Merz W.G.; Wingard, J.R.; Charache, P.; Saral, $R$. Journal of Infections diseases (1980)142(4) 503-509. En inglés. Johns Hopkins ONCOLOGY Center, 601, North Broadway, Baltimore, M.D. 21205, USA.

La vigilancia fúngica de cultivos de orina, deposiciones $y$ de origen respiratorio fueron analiza. dos en 37 receptores de transplantes de médula ósea y 52 pacientes sujetos a quimioterapia por leucemia aguda y otros males hematológicos. Todos los pacientes presentaban una prolongada aplasia. Un $670 \%$ de los pacientes se en. contraban colonizados por Candida albicans y un $28 \%$ o por CANDIDA tropicalis. Se comprobaron 21 casos de micosis sistémica, 3 originadas por Candida albicans, 16 debidas a Candida tropicalis y 2 por Aspergillus. Los datos aportados por la vigilancia positiva para C. tropicalis eran correlacionados con la enfermedad, aspecto que no fue posible correlacionar para el caso de $\mathbf{C}$. albicans.

DETECTION OF CRYPTOCOCCAL ANTIGEN. COMPARISON OF TWO LATEX AGGLUTINATION TESTS.

Kauffman, C.A.; Bergman, A.G.; Severance, P.J.; Mc Clatchey, K.D. American Jornal of Clinical Pathology (1981) 75 (1) 106-109. Inglés, Veterans Admin. Med. Center. Ann. Arbor Michigan 48105, USA.

Un nuevo test comercial mejorado de aglutinación del latex (IBL Kit) fue comparado por el utilizado por el Center for Disease Control (CDC) en cuanto a sensibilidad, especifidad y peso del título antigénico. En un periodo de 13 meses, 335 muestras fueron probadas con ambos kit, hubo solo una falsa reacción $(t)$ con ambos kit $(0,4 \%)$ y una falsa negativa con el CDC kit, entre 18 pacientes afectados por meningitis o infección diseminada por Cryptococcus neoformans. El suero de pacientes que tuvieron criptococosis pul- 
monar localizada, superada, mostraban resultados negativos con ambos kit.

\section{CRYPTOCOCCUS LAURENTII LUNG ABSCESS}

Lynch, J.P.; III; SCHBERG, D.R.; Kissner, D.G.; Kauffman, C.A. American Review of Respiratory Diseases (1981) 123 (1) 135-138. En inglés, Univ. Michigan Hosp. Ann. Arbor, Mich 48109, USA.

Se reporta el caso en una mujer de 55 años afectada de dermatomyositis con tratamiento de corticoesteroides. Este es el primer informe que involucra al Cryptococcus laurentii en una afección pulmonar. La cepa era susceptible "in vitro" a la anfotericina B, pero resistente a la 5 fluorocitosina. No se demostró la existencia de sinergismo. La terapia con anfotericina B $(0,7 \mathrm{mg} / \mathrm{kg})$ junto con la reducción de la dosis de un cortico-esteroides diario dio como resultado la resolución clínica y roentnográfica.

\section{A NOTABLE ASPERGI- LLUS FROM A MORTAL ASPERGILLOMA OF THE LUNG. NEW ASPECTS OF THE EPIDEMIOLOGY. SEROGDIAGNOSIS AND TAXONOMY OF ASPERGILLUS FUMIGATUS}

Staib, F.; Mishra, S.K.; Rajendran, C.; Voigt, R.;Steffen, J.; Neumann K.H.; Hartmann, C.A.; Heins, G. Zentralblatt für Bakterologie und Hygiene, IA (1980) 247(4) 530 536. En inglés. Robert Koch Inst., D-1000 Berlin 65, German Federal Republic.

Se presenta el caso fatal de un varón de 45 años por aspergiloma pulmonar con antecedentes tuberculosos. Los examenes de desgarro y autopsia del pulmón pusieron de manifiesto la existencia de un hongo blanco, de menguado desarrollo típico de Aspergillus fumigatus. Esta cepa blanca produce cabezas conidiales semejantes a $\mathbf{A}$. fumigatus pero con fiálides, aparentemente septadas. Aplicando las técnicas de inmunodifusión y con la participación de ambos antígenos ( 2 cepas) se detectaron anticuerpos y la relación antigénica entre ambos aislamientos. Se presume que la cepa blanca es una variante de A. fumigatus y su presencia señala una aspergilosis crónica o progresiva.

\section{ANTIGENIC ANALYSIS OF TRICHOPHYTON MENTAGROPHYTES COMPLEX}

Ikutomi, $M$.

Japanese Journal of Medical

Mycology (1979) 20(4) 266-272.

En japonés con un resumen en inglés.

Dep. Derm. Koio, Univ. Sch. Med. Tokyo, Japan.

Utilizando métodos inmunolóe inmunoquímicos se estudiaron las propiedades antigénicas de las especies: Arthroderma benhamiae, A. vanbreuseghemii y $T$. interdigitale. El análisis de las componentes de las paredes celulares de las tres especies, indican escasas diferencias entre ellas.

\section{LTF-130. AN EFFECTIVE VACCINE AGAINST BOVINE TRICHOPHY- TOSIS}

Rotermund, $H$

Monatshefte für Veternärmedizin (1980)3519)334-335. Enalemán, Staatl. Tierärzl. Gemeins chaftspraxis, Müggelspree, Bad Saarow, German Democratic Republic.

Los resultados obtenidos durante 40 meses de pruebas, demostraron que la vacuna elaborada en Rusia ha erradicado la infección ocasionada al ganado por Trichophyton verrucosum mediante la aplicación de un programa de vacunación continuada.

Dermatophytes isolated in our clinic of Santiago de Compostela Spain in the last 27 years.

Pereiro, Muguens; M. Ferreirós, Espinoza, $M$.

Mykosen (1980) 23(8) 456-461.

En inglés. Clinic of Santiago de Compostela, Spain.

Los autores aislaron un total de 1.569 dermatofitos. $\mathrm{Si}$ bien es cierto durante su trabajo detectaron una disminución de tinea capitis, pero se incrementaron otras formas clínicas de tiña. Los resultados se presentan a través de un período de 9 años. Las especies aisladas fueron:
- Microsporum audouinii (1)

- Microsporum canis (401)

- Microsporum gypseum (97)

- Trichophyton schoenleinii (82)

- Trichophyton tonsurans (114)

- Trichophyton violaceum (37)

- Trichophyton megninii (4)

- Trichophyton mentagrophytes (261)

- Trichophyton verrucosum (74)

- Trichophyton rubrum (294)

- Trichophyton sp (2)

- Epidermophyton floccosum (202)

\section{SCOPULARIOPSIS} BREVICAULIS IN NAILS Onsberg, $P$.

Dermatologica (1980) 161(4)

259-264. En inglés.

Dep. Derm. Finsten Inst. Copenhagen, Denmark.

De un total de 5.600 pacientes con uñas traumáticas, 226 casos presentaban como agente causal de la lesión la especie Scopulariopsis brevicaulis. Entre 3.700 pacientes con onicomicosis la frecuencia de aislamiento de esta especie como contaminante correspondió a un $2 \%$ de los casos. En 1.925 pacientes las onicomicosis originadas por $\mathbf{S}$. brevicaulis corresponden a un $6 \%$, pero un $10 \%$ de ellas equivalen a infecciones mixtas con la especie Trichophyton rubrum.

\section{THE OPPORTUNISTIC PATHOGENIC FUNGI AND THE REACTION TO THEIR PRESENCE IN THE HUMAN BODY UNDER NORMAL AND PATHOLO- CIAL CONDITIONS \\ Tomšiková, $A$, ; Novácková, $D$. Mykosen (1980) 23(5) 235-258. En alemán \\ Inst. Microbiol. \& Epidemiol., Charles Univ,, Plzen, Czechoslo- vakia.}

Estudio realizado en 9.405 pacientes afectados por enfermedades respiratorias e internas, como también personas sanas. Los hongos semejantes a levaduras, fueron detectados con mayor frecuencia en pacientes con bronconeumonia, neumonia, tuberculosis, con procesos micóticos y en personas debilitadas. En personas sanas, los resultados son generalmente negativos, en tanto que los pacientes con historial alérgico se les detecta 
con mayor frecuencia hongos filamentosos. Las especies más aisladas fueron Candida albicans y Penicillium decumbens. Las reacciones serológicas positivas a Candida se presentaron en enfermos con Candidosis, procesos crónicos $\mathrm{y}$ cáncer.

Las reacciones positivas a Aspergilosis fueron detectadas en pacientes afectados por hongos, individuos comprometidos con terapia de corticoesteroides y trabajadores agrícolas con problemas respiratorios.

THE DOCTOR'S DILEMMA. SYSTEMIC CANDIDIASIS.

Mc Donnell, L.; Farrell, M.D.

Irish Journal of Medical Science (1981) 150 (1) 15-17. En inglés, Departament of Pathology St. Vicent's Hospital, Dublin 4, Irish Republic.

Dos casos fatales de candidosis sistémica se presentaron en pacientes inmunocomprometidos: a) Candida fue aislada del desgarro y orina de un hombre de 60 años con abcesos pericólicos y a partir de hemocultivos; b) de una muestra vaginal y piel de una mujer diabética de 28 años con fractura en ambas piernas. En ambos casos, Candida albicans fue aislada del riñón durante la necropsia. Se entrega un resumen de los métodos de laboratorio sobre los cuales se apoya el diagnóstico de las candidosis sistémica, además de presentar criterios de diagnóstico y lo último en terapia.

\section{THE ACCURATE IDENTI-}

\section{FICATION OF THE YEASTS} OF MEDICAL INTEREST

Douchet, C.; Clauss, B.; Guironnet, J.C.; Muller, J.

Annales Médicales de Nancy et de l'Est (1980) 19 (Mai) 465-468.

En francés, Lab. Biol. Mèd., Hop.

Armées, Limousin, France.

Es posible perfeccionar el diagnóstico de las levaduras aplicando métodos bioquímicos que incluyen 20 características auxonográficas. Se analizaron 602 cepas, de las cuales 228 no pudieron ser identificadas por este sistema, siendo procesadas por el API $20 \mathrm{C}$.
ON THE INCIDENCE OF YEAST SPECIES FROM HUMAN SOURCES IN FINLAND. V. YEAST OCCURRING IN MIXED CULTURES WITH DERMATOPHYTES, OR WITH OTHER YEAST SPECIES. Sonck, C.E. Mykosen (1980) 23(7) 343-350. En inglés. Dep. Derm. Univ. Turku, Finland.

De un total de 54.635 muestras analizadas, se detectaron 16.460 levaduras. El periodo de muestreo abarcó desde 1960 a 1976, observándose que las levaduras y dermatofitos crecían en combinación en 98 cultivos obtenidos de pacientes con tinea cruris. Los dermatofitos identificados fueron: Trichophyton rubrum (53) y Epidermophyton floccosum (35). Entre las levaduras las más frecuentes fueron Pityrosporum ovale (Malassezia ovalis), detectada en un $50 \%$ de cultivos mixtos. La especie Candida albicans concurre con los dermatófitos en un $18 \%$ de los casos.

En los casos de tinea pedis la combinación entre estos dos grupos es más frecuente, Trichophyton mentagrophytes, $T$. rubrum $y$ Epidermophyton floccosum se detectaron asociados a levaduras en 545 casos, las más frecuentes fueron Candida parapsilopsis (141) Torulopsis famata (58) Trichosporon cutaneum (66) (T. beigelii), Candida guillermondii (59) y Candida albicans (57).

También se observó que entre los dedos de los pies de mujeres (168) y hombres (219); en las uñas de mujeres (227) y hombres (79), la concurrencia simultánea de dos especies de levaduras.

\section{THE IDENTIFICATION} OF THE YEASTS BY A SEROLOGICAL METHOD.

Douchet, C.; Muller, $J$. Annales Medicales de Nancy et de l'Est (1980) 19 (Mai) 471-475. En francés, Lab. Biol. Méd. Hop. Armées, Limousin, France.

Usando 11 sueros monoespecíficos, se obtuvo un diagnóstico seguro en el caso de algunas levaduras, en 15 minutos. De un total de 59 levaduras, con esta técnica se identificaron: 28 Torulopsis candida, 6 Candida parapsilopsis, 1 Candida pseudotropicalis, 1 Candida guillermondii, 2 Candida tropicalis, 5 Trichosporon $\mathrm{sp}, 1$ Rhodotorula, 2 Cryptococcus, 2 Saccharomyces y 11 cepas no identificadas. Esta técnica fue comparada en cuanto a rendimiento con los tests bioquímicos $y$ otros de rutina.

\section{MUTAGENICITY AND CARCINOGENICITY OF MYCOTOXINS: DNA BINDING AS A POSSIBLE MODE OF ACTION.}

Stark, A.A.

Annual Review of Microbiology (1980) 34 235-262. Inglés, Dep. Biochem., Tel Aviv Univ. TelAviv, Israel.

El autor revisa la literatura bajo los siguientes encabezamientos: relación entre la actividad genética y carcinogénica de las micotoxinas en general, la actividad genética de aquellas micotoxinas para las cuales no se ha reportado aún carcinogénesis; aflatoxinas y componentes relacionados; relación entre los enlaces del ácido nucleico, carcinogénesis y actividad genética de las micotoxinas. Los compuestos discutidos incluyen la griseofulvina y en su conclusión final el autor recalca y recomienda los pasos a seguir para eliminar las drogas peligrosas como ésta.

\section{TOXIGENIC FUNGI \\ ASSOCIATED WITH}

CEREALS. 1- SURVEY OF

MYCOFLORA ASSOCIATED WITH MAIZE, WHEAT AND RICE.

Salgado, J.M.; Carvalho, P.C.T. De. Revista de Microbiologia (1980) 11(2) 60-63. Portugués, resumen en inglés. Luiz Queiroz Agric. Coll. Piracicaba, SP Brazil.

Los géneros aislados con un mayor porcentaje corresponden a Fusarium, Aspergillus y Diplodia, en menor proporción Penicillium, Acremonium, Curvularia, Trichoderma, y Micelio estéril. 
NEEDED: RESEARCH ON STORAGE MOLDS IN GRAINS SEEDS, AND THEIR PRODUCTS

Christensen, C.M.

Plant Disease (1980)64 (12)10671070. Inglés, Dep. Pl. Path., Univ. Minnesota, St. Paul, USA.

Entre los tópicos que se discuten en este trabajo se destaca el hecho que uno de los requerimientos para la formación de la toxina, es que el productor de la toxina esté presente en el cultivo puro, un requerimiento que rara vez se encuentra en los granos almacenados.

\section{AIR POLUTION WITH FUNGAL SPORES IN A PAPER FACTORY}

Krakowa, $P$.

Bulletin of the International Union aganist Tuberculosis (1979) 54 (2) 190-191. En inglés. Inst. Tuberculosis, ul. Plocka 26, 01-138. Warsow Poland.

Este estudio está basado en una investigación llevada a cabo en los meses de mayo a noviembre de 1975 en una factoría de papel que procesa la madera de pino. Aspergillus fumigatus fue la especie predominante en todas las muestras. También se detectó Penicillium sp. De los 222 trabajadores examinados sólo uno tenia precipitinas contra Aspergillus fumigatus, pero sin sintomas de alveolitis alérgica. Este hallazgo sugiere que los frecuentes cambios de ocupación y de lugar físico de trabajo dentro de la factoría, disminuyen el riesgo de contraer una alveolitis alérgica.

\section{Drugs for systemic fungal} infections

Stevens, D.A.

Rational Drug Therapy (1979)

13(5) 1-7. En inglés, Dep. Med. Sta. Clara Valley Med. Center,

San José, California. USA.

Entre las drogas revisadas se cuent an 1a anfotericina $B$, la 5 fluorocitosin a (ambos en combinación) y miconazol. 